\title{
Efforts towards a low-temperature-sensitive physics package for vapor cell atomic clocks
}

\author{
Qiang Hao ${ }^{1 *} \mathbb{0}$, Wenxiang Xue ${ }^{1,2}$, Feng $X^{3}{ }^{3}$, Kemu Wang ${ }^{1,2}$, Peter Yun ${ }^{1,2}$ and Shougang Zhang ${ }^{1,2}$
}

\begin{abstract}
Strong environmental dependence is an intractable problem for vapor cell clocks, for which the high-temperature sensitivity of the physics package is considered one of the dominant reasons. In this paper, we report the design and realization of a low-temperature-sensitive physics package for vapor cell clocks. The physics package comprises three layers of magnetic shields, three layers of heating ovens, and the cavity-cell assembly. The cavity-cell assembly employs a compact magnetron-type cavity and a Rb vapor cell sealed with $\mathrm{N}_{2}$-Ar mixed buffer gas. The dependence of the clock frequency on temperature fluctuation is evaluated to be $2 \times 10^{-11} /{ }^{\circ} \mathrm{C}$. In pursuit of the stable temperature, a three-stage temperature regulator is implemented on the physics package. It adopts a combination of open andclosed-loop control to address the problem of significant thermal coupling between the heating ovens. Under a laboratory environment, the measured Hadamard deviation of the temperature variation is $4 \times 10^{-5}{ }^{\circ} \mathrm{C}$ in 1 day of averaging.
\end{abstract}

Keywords: Vapor cell atomic clock, Physics package, Temperature sensitivity

\section{Introduction}

In recent years, vapor cell atomic clocks, which play an important role in Global Navigation Satellite Systems (GNSSs), have attracted considerable attention (Camparo 2007; Godone et al. 2015). It is reported that lamppumped $\mathrm{Rb}$ clocks operating in vacuum present the long-term stability of $3 \times 10^{-15}$ (Vannicola et al. 2010; Mei et al. 2016). Additionally, vapor cell clocks in different configurations, including lamp-pumped Rb clock (Hao et al. 2016), continuous-wave laser-pumped Rb clock (Bandi et al. 2014), pulsed optically pumped (POP) $\mathrm{Rb}$ clock (Micalizio et al. 2012), and coherent population trapping (CPT) Cs clock (Abdel Hafiz et al. 2018), have shown excellent short-term (less than $100 \mathrm{~s}$ averaging time) stabilities better than $3 \times 10^{-13} \mathrm{~T}^{-1 / 2}$. It is noteworthy that the shot noise limitations of the POP Rb clock and the CPT Cs clock are far beyond their measured performances.

\footnotetext{
*Correspondence: haoq@ntsc.ac.cn

${ }^{1}$ Key Laboratory of Time and Frequency Primary Standards, National Time Service Center, Xi'an 710600, People's Republic of China

Full list of author information is available at the end of the article
}

As is well known, the buffer gases in vapor cell are to narrow the resonance signal and quench the excited atoms. The gases shift the clock's resonant frequency by a few $\mathrm{kHz}$, which is sensitive to temperature fluctuation. Usually, the frequency stability of vapor cell clocks tend to degrade when the averaging time is larger than $1000 \mathrm{~s}$ in air. To obtain a better long-term $(10,000 \mathrm{~s}$ or even longer) frequency stability, the vapor cell clocks are generally placed in the vacuum chamber or the thermostat for ground-based applications. This strategy increases the cost and size of vapor cell clocks.

The existing high-performance vapor cell clocks are mainly designed for space-based applications. Researches focusing on the long-term performance in air are limited (Moreno et al. 2018; Almat et al. 2020). As the temperature sensitivity of the physics package has been identified as a major limitation for long-term stability (Micalizio et al. 2012; Calosso et al. 2012), the primary aim of this study is to realize a low-temperature-sensitive physics package for high-performance vapor cell clocks, which could benefit the ground-based applications, including GNSS user terminal, very long baseline interferometry (VLBI), and telecommunication. 


\section{Cavity-cell assembly design}

Some vapor cell clocks require a microwave cavity to select the resonant mode of the microwave field. A standard cylindrical microwave cavity with $\mathrm{TE}_{011}$ mode is employed in a POP Rb clock (Godone et al. 2011), which has a typical volume of $100 \mathrm{~mL}$. For such a cavity, the vapor cell is held in the core of the cylindrical cavity by long stems, which makes it difficult to stabilize the cell temperature via the heat conduction of the glass stems. Conversely, the magnetron cavity has a small size, and the resonant mode $\mathrm{TE}_{011}$-like permits the magnetic component to be parallel to the quantization axis across the cavity. These characteristics enable the direct glue of the vapor cell on the metal cavity's inner wall, which enhances the heat transfer between the vapor cell and heating oven. As shown in Fig. 1, a magnetron cavity includes two cylindrical shells, and the inner one is divided by several equally spaced slots. With the lumped elements equivalent model, the slots act as the capacitance $C$ (Froncisz and Hyde 1982; Stefanucci et al. 2012):

$$
C=\frac{\varepsilon w h}{t n}
$$

where $\varepsilon$ is the dielectric constant, and $w, h, t, n$ are the thickness, height, width, and number of slots, respectively. Likewise, the metal electrodes act like the inductance $L$ :

$$
L=\frac{\mu_{0} \pi r^{2}}{h}
$$

where $\mu_{0}$ is the permeability of vacuum and $r$ is the radius of the inner shell. Considering the frequency shift generated by the outer shell and fringing fields, the resonant frequency $v$ is given by

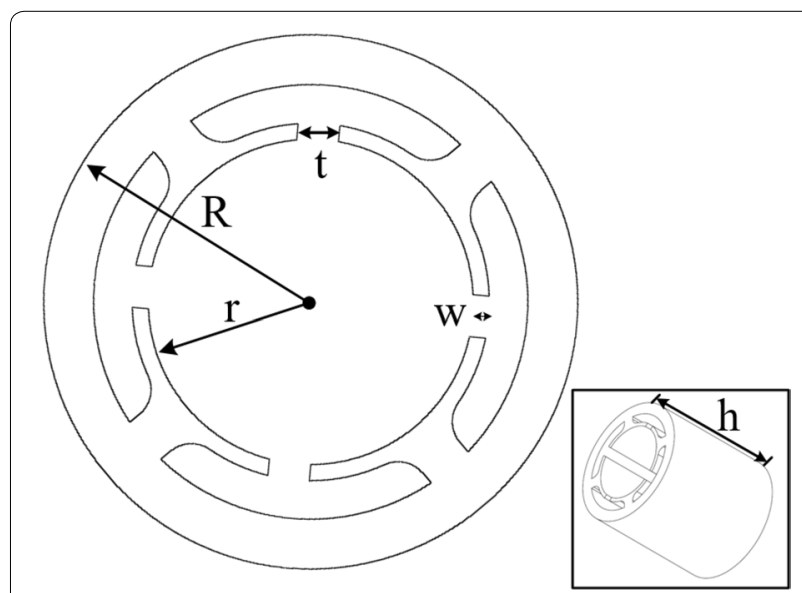

Fig. 1 Schematic of the magnetron-type microwave cavity

$$
\begin{aligned}
v= & \frac{1}{2 \pi r}\left(\frac{n t}{\pi w \varepsilon_{0} \mu_{0}}\right)^{1 / 2}\left(1+\frac{r^{2}}{R^{2}-(r+w)^{2}}\right)^{1 / 2} \\
& \left(\frac{1}{1+2.5(t / w)}\right)^{1 / 2}
\end{aligned}
$$

where $R$ is the outer shield radius. Hence, the resonant frequency can be easily adjusted by changing the number $n$, thickness $t$, and width $w$ of the slots.

As shown in Fig. 2a, we designed a copper magnetrontype cavity with four electrodes. It has an external diameter of $33 \mathrm{~mm}$ and a height of $35 \mathrm{~mm}$; thus, its volume is approximately one third of the standard $\mathrm{TE}_{011}$ cavity. The microwave signal is transmitted with the antenna through a microwave cable and couples into the cavity. With the high frequency structure simulator (HFSS) software, Fig. $2 \mathrm{~b}$ shows the numerical simulation of the magnetic field orientation inside the cavity. The magnetic lines are highly parallel to each other, indicating that the resonant mode is $\mathrm{TE}_{011}$-like. Additionally, the measured Zeeman transition strengths between the hyperfine levels $\mathrm{F}=1$ and $\mathrm{F}=2$ of ${ }^{87} \mathrm{Rb}$ ground-state are shown in Fig. 2c, and the deduced field orientation factor is as high as 0.9 (Stefanucci et al. 2012). The loaded quality factor measured 120. Based on a research on a magnetron-type cavity with a loaded quality factor of 140 (Almat et al. 2020), we believe that the cavity-pulling effect caused by cavity temperature variations is negligible for such a cavity.

To characterize the performance of the proposed cavity, the clock signal is measured with a POP Rb clock. A contrast of Ramsey central fringe of $31 \%$ is obtained when the temperature of the vapor cell is set to $65^{\circ} \mathrm{C}$, as shown in Fig. 2d. The linewidth of the central fringe is $148 \mathrm{~Hz}$, which is consistent with the theoretical value 1/ (2T) (Ramsey time $\mathrm{T}=3.5 \mathrm{~ms}$ ). Additionally, the calculated shot noise limit of the package is $1.6 \times 10^{-14} \tau^{-1 / 2}$, demonstrating no signal to noise ratio loss compared to previous works (Micalizio et al. 2012; Kang et al. 2015).

The primary reason for the high-temperature sensitivity is the collision shift between $\mathrm{Rb}$ atoms and buffer gases. An empirical second-order polynomial expression for this effect is (Vanier et al. 1982)

$$
\Delta f=P_{t}\left(\delta^{\prime} \Delta T+\gamma^{\prime} \Delta T^{2}\right)
$$

where $\Delta f$ is the temperature-depended frequency shift, $P_{t}$ is the total gas pressure, $\delta_{0}$ and $\gamma_{0}$ are the linear and quadratic temperature coefficients, respectively, and $\Delta T$ is the difference between the operating temperature and reference temperature. Here, we used a vapor cell of $20 \mathrm{~mm}$ length and $20 \mathrm{~mm}$ external diameter. It was filled with a mixture of $\mathrm{Ar}$ and $\mathrm{N}_{2}\left(\mathrm{P}_{\mathrm{Ar}} / \mathrm{P}_{\mathrm{N} 2}=1.6\right)$ with a total pressure of 25 Torr. The temperature coefficient is measured and 
$\mathbf{a}$
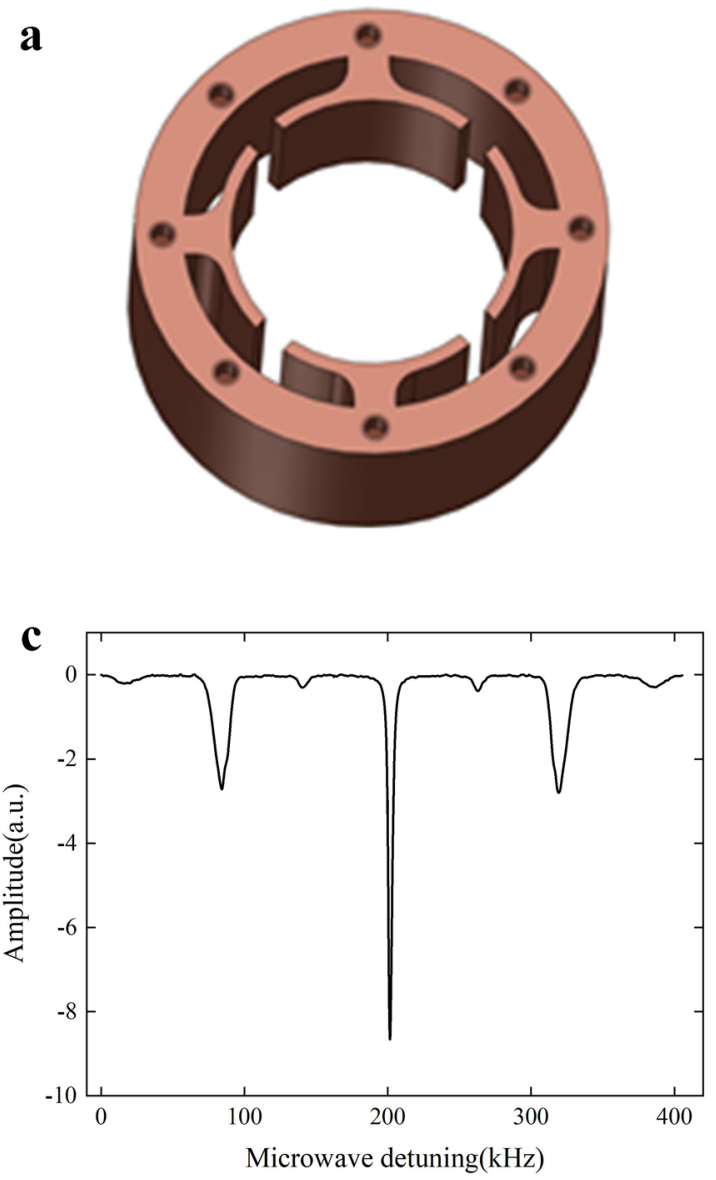

b
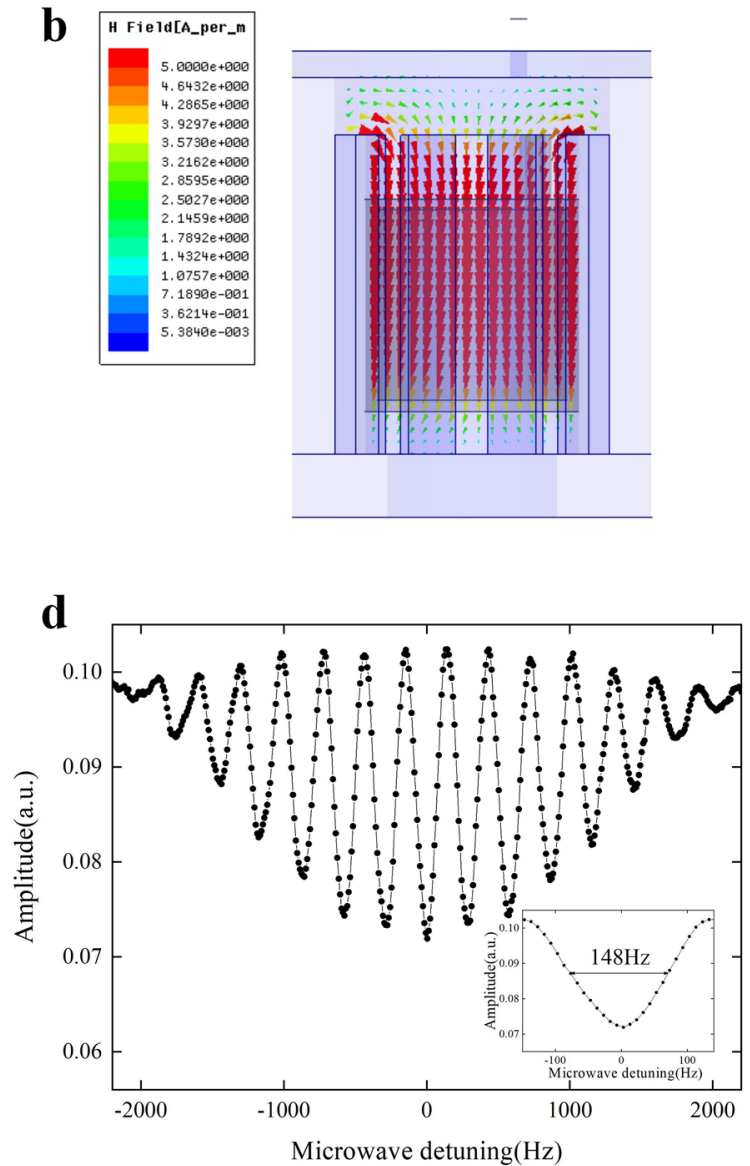

Fig. 2 a Structure of the magnetron-type cavity, $\mathbf{b}$ Simulated result of the microwave magnetic field with a glass cell inside, $\mathbf{c}$ Zeeman transition strengths between the hyperfine levels $\mathrm{F}=1$ and $\mathrm{F}=2$ of ${ }^{87} \mathrm{Rb}$ ground state, $\mathbf{d}$ The observed Ramsey fringe. The sequence of the Ramsey interrogation is set as follows: pumping pulse duration $T_{p}=0.4 \mathrm{~ms}$, microwave pulse duration $T_{\mu}=0.4 \mathrm{~ms}$, Ramsey time $\mathrm{T}=3.5 \mathrm{~ms}$, and optical detecting pulse duration $T_{d}=0.2 \mathrm{~ms}$
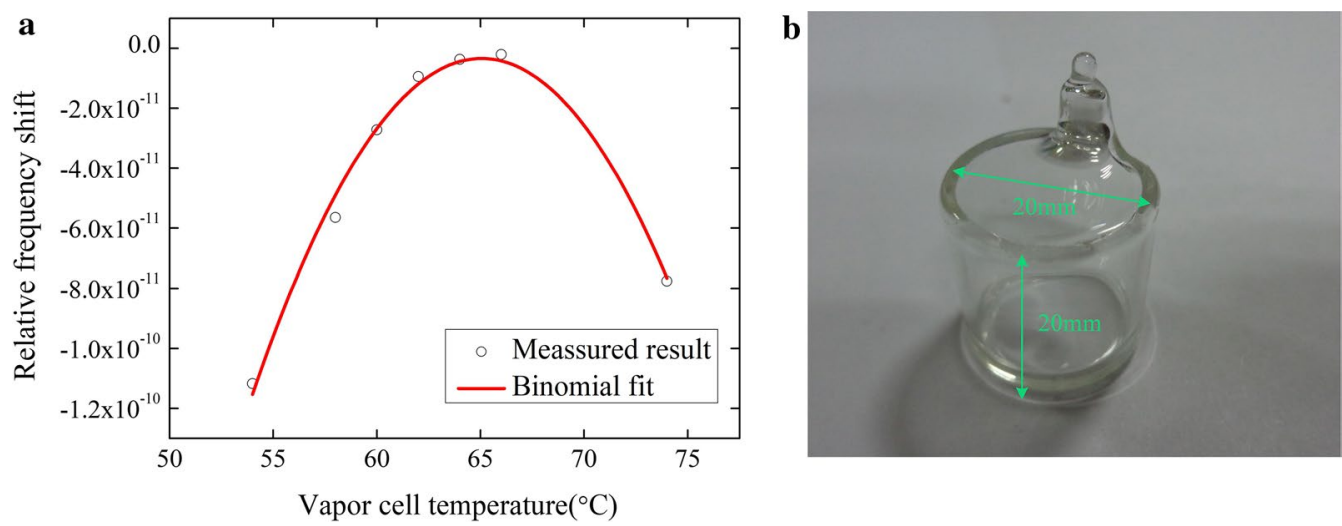

Fig. 3 Relative frequency shift with temperature (a) and photo (b) of the gas cell

shown in Fig. 3a. The result is well-fitted by the binomial fit. From Fig. 3a, we conclude that the relative frequency shift is at a negligible level of $10^{-12} /{ }^{\circ} \mathrm{C}$ when setting the cavity temperature about $65^{\circ} \mathrm{C}$.
The geometric effect of the vapor cell also yields an enhanced temperature coefficient (ETC) (Calosso et al. 2012). As shown in Fig. 3b, a cell stem with $1 \%$ volume of the total is placed in the cavity. As the temperature 
fluctuation is small enough inside the cavity, it is unnecessary to stabilize the temperature of the stem independently. The temperature gradient produced by the heat conduction of glass is designed to avoid the $\mathrm{Rb}$ migration effect, and the ETC is theoretically estimated to be as small as $2 \times 10^{-11} /{ }^{\circ} \mathrm{C}$ in our case.

\section{Thermal design and temperature control}

Temperature control is of importance for mitigating the temperature sensitivity. The structure of the package is shown in Fig. 4. It includes three layers of magnetic shields, three layers of heating ovens, and the cavity-cell assembly. The Helmholtz coils are wrapped on the surface

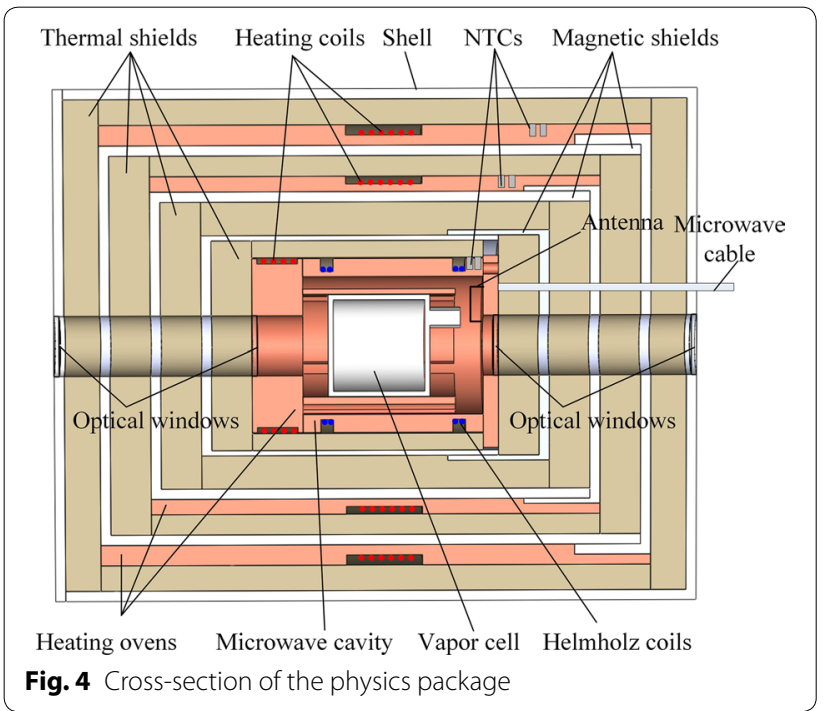

of the cavity, and the heating coils for the inner heating oven are wrapped on the surface of copper cutoff waveguide. The cavity-cell assembly is housed in three layers of magnetic shield. The middle and outer magnetic shields are surrounded by the middle and outer heating ovens, respectively. The rest space excluding the optical path of the package is filled with a polyurethane material as the passive insulators. The thermal conductivity and heat capacity of polyurethane are $0.02 \mathrm{~W} \mathrm{~m}^{-1} \mathrm{~K}^{-1}$ and $1.4 \mathrm{~J} /$ $\mathrm{kg} \mathrm{K}$, respectively. Furthermore, the cavity-cell assembly and the magnetic shields are orderly screwed in place from inside to outside. The sensors and heaters' wires and the microwave cable are placed in several narrow apertures. Although those small holes could degrade the thermal isolation, the three-stage temperature control still enables a small temperature fluctuation (see the details below). The optical detector (PDA 36A) is placed after the physics package to record the optical absorption of the vapor cell.

Attention should also be directed toward the thermal convection enhanced by the laboratory's ventilation system. As shown in Fig. 4, four optical windows are set in the package to reduce the thermal convection in optical propagation direction. Regarding the thermal radiation, an aluminum shell is used because polyurethane has a larger radiation coefficient. Owing to the compact microwave cavity, the size of the new package is approximately $1 \mathrm{~L}$, which is acceptable for the high-performance vapor cell clocks with the volume of several liters. The equivalent electrical model with the lumped parameters of the thermal control for the physics package is shown in Fig. 5. The capacitance $C$ is calculated by $C=c_{0} \rho V$, where $c_{0}$ is the heat capacitance, $\rho$ the density, and $V$

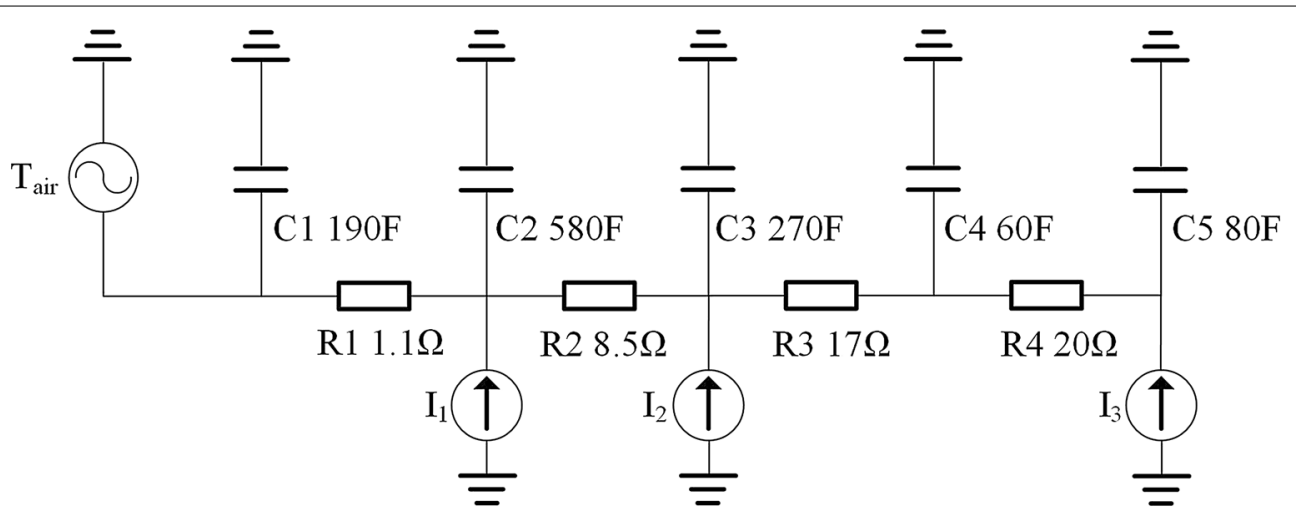

$\mathrm{T}_{\text {air }}$ : Air, C1: Shell, R1:Thermal insulator 1, C2:Magnetic sheild 1 and Thermal oven

1, R2:Thermal insulator 2, C3: Magnetic shield 2 and Thermal oven 2, R3: Thermal

insulator 3, C4: Thermal insulator 3, R4: Thermal insulator 4, C5: Cavity-cell assembly

Fig. 5 Equivalent circuit of the temperature control of the physics package 
the volume. The resistance $R$ is calculated by $R=L /(\lambda S)$, where $L$ is the length, $\lambda$ the conductivity, and $S$ the area. Note that the capacitances of the thermal insulators and the resistances of the metal parts are negligible and thus play negligible roles in this multi-order low pass filter. The equivalents of the three heaters are the three current sources, i.e., $I_{1}, I_{2}$, and $I_{3}$. Thus, the calculated thermal time constant is $76,000 \mathrm{~s}$.

Moreover, heating ovens are necessary as the vapor cell operates at $40{ }^{\circ} \mathrm{C}$ above room temperature. One or two heating ovens serving as the active low pass filters are typically used in the physics package. As the higher-order filter can increase the roll-off and decrease the cutoff frequency (Ogata 2010), we adopt three layers of heating ovens. However, we find that if the three heating ovens are driven simultaneously by the proportional-integral (PI) controllers, the optimization of the parameters, such as proportional gain, integration time, and heating power, are complicated. This is likely due to the significant thermal coupling between the heating ovens.

We replace the driver for the middle heating oven with an open-loop controller, i.e., voltage source, the outer and the inner heating ovens remain driven by the closed-loop controllers, i.e., PI controllers. This method can reduce the thermal coupling between the inner heating and outer heating ovens effectively, and thus make it easy to optimize the parameters of the PI controllers. The PI temperature regulation is similar to the previous work (Esman and Rode 1983), but several modern low-temperature coefficient electronic components are used in this study. Owing to the large fractional resistance variation $\left(700 \Omega /{ }^{\circ} \mathrm{C}\right.$ at $\left.65^{\circ} \mathrm{C}\right), 100 \mathrm{k} \Omega$ resistors with Negative Temperature Coefficient (NTC) are used as the in-loop and out-of-loop thermal sensors. The influences of the wire and thermistor self-heating accordingly become negligible. As shown in Fig. 4, the monitoring and feedback NTCs are placed nearly at the same place inside each heating oven. The

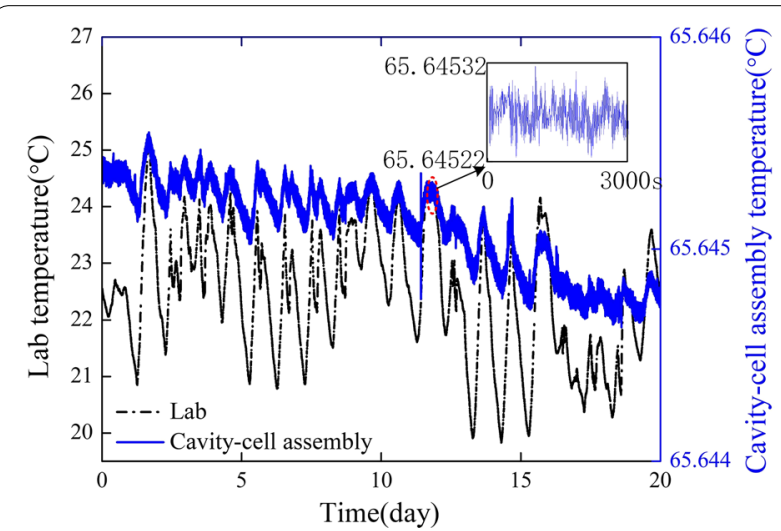

Fig. 6 Recordings of the cavity-cell assembly and laboratory temperature. Inset: short-term fluctuation of the cavity-cell assembly

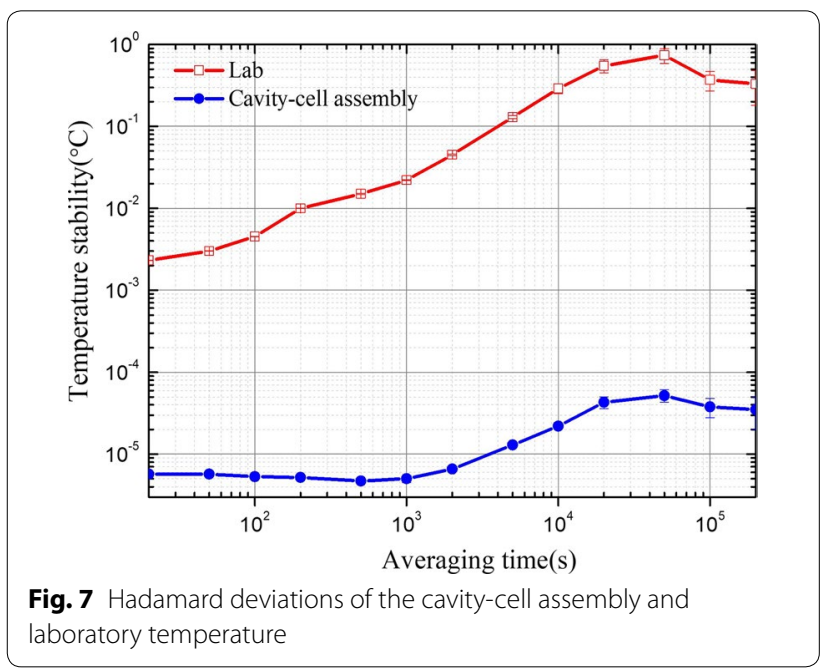

error signals are converted to voltage with a bridge, in which three resistors (Vishay Z201) with $0.2 \mathrm{ppm} /{ }^{\circ} \mathrm{C}$ temperature coefficient are used as the reference. The bridge is driven by a DC reference (LM 399) with $0.5 \mathrm{ppm} /{ }^{\circ} \mathrm{C}$ temperature coefficient. The voltage source used for the openloop control is a commercial product (GWINSTEK GPS 330C).

Temperature variation of the physics package is tested in a laboratory with the cycled room-temperature variation of $\sim 4{ }^{\circ} \mathrm{C}$. The NTC for measuring laboratory temperature is recorded with a Keithley digital multimeter 2701 with 6.5 digits of resolution and $20 \mathrm{ppm} /{ }^{\circ} \mathrm{C}$ temperature coefficient. The NTC for monitoring temperature of the cavity-cell assembly is recorded by a Keysight multimeter 3458A with 8.5 digits of resolution and $3 \mathrm{ppm} /{ }^{\circ} \mathrm{C}$ temperature coefficient. As shown in Fig. 6, the temperature of the cavity-cell assembly demonstrates the excellent short-term fluctuation of less than $0.1 \mathrm{~m}{ }^{\circ} \mathrm{C}$, and the 1-day fluctuation of $\sim 0.5 \mathrm{~m}^{\circ} \mathrm{C}$ during the 20-day measurement. The drift of the cavity-cell assembly temperature is also as small as $20 \mu^{\circ} \mathrm{C}$ per day. As the longterm frequency stability of a vapor cell clock is generally characterized by Hadamard deviation, the Hadamard deviations of the cavity-cell assembly and laboratory temperature are calculated and shown in Fig. 7. It can be found the temperature stability of the cavity is about $6 \times 10^{-6}{ }^{\circ} \mathrm{C}$ for the averaging times from 20 to $1000 \mathrm{~s}$. This indicates that the short-term stability is mainly impacted by the flicker frequency noise originated from the temperature controller. The temperature stability for the averaging times between 1000 and 20,000 s increases from $6 \times 10^{-6}$ to $5 \times 10^{-5}{ }^{\circ} \mathrm{C}$, which is consistent with the variation of laboratory temperature determined originated from the daily cycled ambient temperature. From Fig. 7, one can also find that the day temperature stability of the cavity-cell assembly reaches $4 \times 10^{-5}{ }^{\circ} \mathrm{C}$, which is 
comparable to the result with the help of vacuum chamber (Micalizio et al. 2012).

\section{Conclusions}

We investigate a physics package with a low temperature coefficient and stable temperature. Among the temperature-related effects such as buffer-gas induced frequency shift and cavity pulling, the ETC is found to be the major contribution to the long-term instability. With the evaluated ETC of $2 \times 10^{-11} /{ }^{\circ} \mathrm{C}$ and cell temperature stability of $4 \times 10^{-5}{ }^{\circ} \mathrm{C}$, the contribution of the ETC to long-term stability is only $8 \times 10^{-16}$ for 1 day averaging time. Moreover, the variation of the environmental temperature of $4{ }^{\circ} \mathrm{C}$ indicates our physics package doesn't require the moderate operating condition. Since studies (Micalizio et al. 2012; Almat et al. 2020) have demonstrated a longterm frequency stability better than $1 \times 10^{-14}$ when placing the physics package either in a vacuum chamber or under stable pressure condition and exposing other modules to air, the techniques presented in this study could help in improving the performance of vapor cell clocks in term of ground-based applications.

\section{Abbreviations}

GNSS: global navigation satellite system; POP: pulsed optically pumped; CPT: coherent population trapping; VLBI: very long baseline interferometry; HFSS: high frequency structure simulator; ETC: enhanced temperature coefficient; PI: proportional-integral; NTC: negative coefficient characteristic.

\section{Acknowledgements}

The authors thank D. Li for careful reading of this manuscript. Q. Hao thanks Z. Du for the help of vapor cell fabrication.

\section{Authors' contributions}

$\mathrm{QH}$ and SZ conceived the presented idea; $\mathrm{QH}, \mathrm{WX}, \mathrm{FX}, \mathrm{PY}$, designed the temperature controller; $\mathrm{QH}, \mathrm{WX}, \mathrm{KW}$ carried out the experiment and analyzed the results; $\mathrm{QH}$ wrote the manuscript. All authors read and approved the final manuscript.

\section{Funding}

This work is supported in part by the National Natural Science Foundation of China under Grant No. 11703031, U1731132 and in part by the West Light Foundation of the Chinese Academy of Sciences under Grant No. XAB2017B04.

\section{Availability of data materials}

Not applicable.

\section{Ethical approval and consent to participate}

Not applicable.

\section{Consent for publication}

The authors declare that they consent to the publication of this work.

\section{Competing interests}

The authors declare that they have no competing interests.

\footnotetext{
Author details

${ }^{1}$ Key Laboratory of Time and Frequency Primary Standards, National Time Service Center, Xi'an 710600, People's Republic of China. ${ }^{2}$ University of Chinese Academy of Sciences, Beijing 100049, People's Republic of China.
}

${ }^{3}$ China Academy of Space Technology (Xi'an), Xi'an 710600, People's Republic of China.

Received: 13 September 2019 Accepted: 7 April 2020

Published online: 25 May 2020

\section{References}

Abdel Hafiz, M., Coget, G., Petersen, M., Rocher, C., Guérandel, S., Zanon-Willette, T., et al. (2018). Toward a high-stability coherent population trapping Cs vapor-cell atomic clock using autobalanced ramsey spectroscopy. Physical Review Applied, 9, 064002.

Almat, N., Gharavipour, M., Moreno, W., Gruet, F., Affolderbach, C., \& Mileti, G. (2020). Long-term stability analysis toward < 10-14 level for a highly compact POP Rb cell atomic clock. IEEE Transactions on Ultrasonics, Ferroelectrics, and Frequency Control, 67(1), 207-216.

Bandi, T., Affolderbach, C., Stefanucci, C., Merli, F., Skrivervik, A. K., \& Mileti, G. (2014). Compact high-performance continuous-wave double-resonance rubidium standard with $1.4 \times 10^{-13} \mathrm{~T}^{-1 / 2}$ stability. IEEE Transactions on Ultrasonics, Ferroelectrics, and Frequency Control, 61(11), 1769-1778.

Calosso, C. E., Godone, A., Levi, F., \& Micalizio, S. (2012). Enhanced temperature sensitivity in vapor-cell frequency standards. IEEE Transactions on Ultrasonics, Ferroelectrics, and Frequency Control, 59(12), 2646-2654.

Camparo, J. (2007). The rubidium atomic clock and basic research. Physics Today, 60(11), 33-39.

Esman, R., \& Rode, D. (1983). 100- $\mu$ K temperature controller. Review of Scientific Instruments, 54, 1368-1370.

Froncisz, W., \& Hyde, J. S. (1982). The loop-gap resonator: a new microwave lumped circuit ESR sample structure. Journal of Magnetic Resonance, 47(3), 515-521.

Godone, A., Levi, F., Calosso, C. E., \& Micalizio, S. (2015). High-performing vaporcell frequency standards. Rivista Del Nuovo Cimento, 38(3), 133-171.

Godone, A., Micalizio, S., Levi, F., \& Calosso, C. (2011). Microwave cavities for vapor cell frequency standards. Review of Scientific Instruments, 82(7), 074703.

Hao, Q., Li, W., He, S., Lv, J., Wang, P., \& Mei, G. (2016). A physics package for rubidium atomic frequency standard with a short-term stability of $2.4 \times 10^{-13} \tau^{-1 / 2}$. Review of Scientific Instruments, 87(12), 123111.

Kang, S., Gharavipour, M., Affolderbach, C., Gruet, F., \& Mileti, G. (2015). Demonstration of a high-performance pulsed optically pumped Rb clock based on a compact magnetron-type microwave cavity. Journal of Applied Physics, 117(10), 460-908.

Mei, G., Zhong, D., An, S., Zhao, F., Qi, F., Wang, F., et al. (2016). Main features of space rubidium atomic frequency standard for BeiDou satellites. In 2016 European Frequency and Time Forum (EFTF), York, UK, 2016 (pp. 0-3).

Micalizio, S., Calosso, C. E., Godone, A., \& Levi, F. (2012). Metrological characterization of the pulsed Rb clock with optical detection. Metrologia, 49, $425-436$.

Moreno, W., Pellaton, M., Affolderbach, C., \& Mileti, G. (2018). Barometric effect in vapor-cell atomic clocks. IEEE Transactions on Ultrasonics, Ferroelectrics, and Frequency Control, 65(8), 1500-1503.

Ogata, K. (2010). Modern control engineering (5th ed.). Upper Saddle River: Prentice Hall.

Stefanucci, C., Bandi, T., Merli, F., Pellaton, M., Affolderbach, C., Mileti, G., et al. (2012). Compact microwave cavity for high performance rubidium frequency standards. Review of Scientific Instruments, 83(10), 104706.

Vanier, J., Kunski, R., Cyr, N., Savard, J. Y., \& Têtu, M. (1982). On hyperfine frequency shifts caused by buffer gases: Application to the optically pumped passive rubidium frequency standard. Journal of Applied Physics, 53(8), 5387-5390.

Vannicola, F., Beard, R., White J, Senior, K., Largay, M., \& Buisson, J. (2010). GPS block IIF atomic frequency standard analysis. In Proceedings of the 42nd annual precise time and time interval (PTTI) systems and applications meeting, Reston, Virginia (pp. 181-196).

\section{Publisher's Note}

Springer Nature remains neutral with regard to jurisdictional claims in published maps and institutional affiliations. 